\title{
Olfm4 Is Highly Expressed In Liver Cancer Patients And As A Biomarker And Therapeutic Target For Liver Cancer
}

Qingzhu Song ( $\nabla$ songqingzhu5566@163.com )

Bayannur Hospital https://orcid.org/0000-0003-2055-7300

\section{Research Article}

Keywords: OLFM4, biomarker, therapeutic targets, diagnosis, liver cancer

Posted Date: July 13th, 2021

DOl: https://doi.org/10.21203/rs.3.rs-686335/v1

License: (c) (i) This work is licensed under a Creative Commons Attribution 4.0 International License.

Read Full License 


\section{Abstract}

Liver cancer is one of important cancer types causing a large number death in the world, and the incidence is still increasing. Conventional therapies against liver cancer are not satisfied and pathogenesis of liver cancer remains unclear. Thus, the more effective therapies are needed to treat liver cancers, and the discovery of key genes involving in pathogenesis of liver cancers is important for developing more effective therapies to treat liver cancer. In the present study, we found that OLFM4 blood level is higher in liver cancer patients than in healthy individuals, and mRNA expression level in liver cancer tissue than in liver paracancerous tissues. OLFM4 has high predictive capacity as a biomarker for liver cancer and closely correlated to tumor size. Importantly, it is confirmed that OLFM4 contributes to cancer cell proliferation, and HIF-1a involves in this activity. We believe that OLFM4/HIF-1a axis might be a target signaling pathway for developing novel drugs to treat liver cancer.

\section{Introduction}

Liver cancer has been the fourth leading cause of cancer death after lung, colorectal, and stomach cancer, and the incidence is still increasing in the world [1]. For liver cancer, the most common type is hepatocellular carcinoma, followed by cholangiocarcinoma [2]. The pathogenesis of liver cancer is complicated, among them, infections including hepatitis B virus (HBV), hepatitis $\mathrm{C}$ virus (HCV), behavioral factors (alcohol, tobacco), metabolic factors (excess body fatness), and aflatoxins are thought to be the major risks [3]. In general, chemotherapy and immunotherapy are the mostly used in treating hepatocellular carcinomas (HCCs) [4]. For the more advanced stages of liver cancers,trans-arterial chemoembolization (TACE) is often used, which is reported to reach a $23 \%$ improvement in the 2-year survival compared with the conservative therapy for intermediate stage HCC patients [5]. As a kinase inhibitor, the oral format of sorafenib is also often used in the late-stage liver cancers, while its effects are far away from satisfactions and long-term utilization of the drug may cause toxicity and/or drug inefficacy [6]. Moreover, the prognosis of the liver cancer is low; resulting in that only low ratio of liver cancer patients is eligible for surgical removal [6]. Therefore, the more effective therapies are needed to treat liver cancers. Importantly, the discovery of key genes involving in pathogenesis of liver cancers plays an important role in developing novel therapies treating liver cancers.

OLFM4 (olfactomedin 4) belongs to the olfactomedin family, which is also known as hGC-1 (human granulocyte colony-stimulating factor-stimulated clone 1) [7]. Initially, OLFM4 was found to regulate inflammatory response and innate immunity [8]. Moreover, as a secreted protein OLFM4 is closely involved in a variety of cellular functions including proliferation, differentiation, apoptosis, and cell adhesion [9]. Recently, accumulating evidence confirming that OLFM4 plays an important role in regulating growth and proliferation of several types of cancer cells [9]. In esophageal adenocarcinoma, it was found that OLFM4 is closely linked to the nodal metastases [10]. The expression level is found to significantly increased in intestinal metaplasia (IM), while it is absent in normal gastric mucosa [11]. In terms of metastatic breast cancer, it was found that the expression level of OLFM4 is remarkably associated to pathological T factor, distant metastasis and Ki67 status in the ER-positive breast 
carcinomas [12]. OLFM4 is thought to be a potential biomarker for gastrointestinal cancers [13]. In fact, OLFM4 serum level has been biomarker of a variety of diseases including asthmatics [14], non-small cell lung cancer [15], pancreatic, head and neck, and prostate cancers [16]. However, effects of OLFM4 in liver cancers remain unclear, which needs more investigations.

OLFM4/HIF-1a axis was found to involve in regulation of hypoxia-induced invasion, epithelialmesenchymal transition, and chemotherapy resistance in non-small-cell lung cancer [17]. HIF-1a is a subunit of a heterodimeric transcription factor hypoxia-inducible factor 1 (HIF-1), which exerts an essential role in cellular response to systemic oxygen levels in mammals [17]. HIF-1a closely involves in pathogenesis of cancers. HIF-1 $a$ and GATA3 forms a complex to enhance cancer cell invasiveness [18]. Targeting HIF-1a is considered to be a potential therapy for alleviating chemoresistance to enhance the efficacy of chemotherapies in colon cancers [19]. Interestingly, it was found that inhibition of OLFM4/HIF1a axis could improve hypoxia-induced invasion, epithelial-mesenchymal transition, and chemotherapy resistance of non-small-cell lung cancer [17]. Therefore, in the study, we first measured the blood level of OLFM4 in the healthy control and liver cancer patients, and OLFM4 RNA and protein level in liver paracarcinoma tissue and tumor. We confirmed that OLFM4 might be a potential biomarker for liver cancer diagnosis with high sensitivity and specificity. Finally, we confirmed that silencing OLFM4 could reduce liver cancer cell proliferation, which was through targeting HIF-1a. Herein, the study proposes OLFM4 as a potential biomarker and therapeutic target for liver cancer, which provides important information for studying pathogenesis and developing novel drugs against liver cancer.

\section{Materials And Methods}

\section{Patients selection}

In the study, we recruited 100 liver cancer patients and 100 healthy controls, from Bayannur Hospital, from May 2016 to May 2020. Liver cancer patients were diagnosed using histopathological analysis. Among 100 liver cancer patients, 80 received surgery, and 20 patients underwent interventional therapy. Liver cancer was diagnosed according to immunohistochemistry according to the AASLD guide lines. Patients who received radiotherapy or had a history of other solid tumors were excluded. NASH was confirmed based on histopathology of liver biopsy samples, and supported by imaging evidences such as CT and liver ultrasound. Chronic HBV infection was confirmed by HBsAg presence for the last 6 months with an HBV DNA concentration to $>1 \times 10^{3}$ copies per $\mathrm{mL}$ as well as abnormal concentration of serum alanine amino transferase. Confirmation of chronic HCV infection is used qualitative HCV RNA measurement, and more than $1 \times 103$ copies of HCV-RNA in the serum were confirmed to be positive. Healthy controls were identified as without liver or other systematic diseases, or HBV markers (HBsAg, $\mathrm{HBeAg}$, anti-HBe, and anti-HBc), as well as normal concentrations of liver function enzymes. The study was approved by the institutional ethics review committee at Bayannur Hospital. Informed consents were obtained from all participants based on each committee's regulations. Informed written consent was obtained from all patients. 


\section{Serum samples}

Serum samples were obtained from patients who were diagnosed with primary liver cancer at Bayannur Hospital. Serum from healthy individuals were simultaneously collected at Bayannur Hospital as control samples. Serum samples were collected under institutional approval. The serum was centrifuged, aliquoted and stored at $-80^{\circ} \mathrm{C}$ for diagnosis utilization.

\section{Enzyme linked immunosorbent assay}

The concentrations of OLFM4 were detected using ELISA. ELISA kits were purchased from Abcam (catalog number: ab267805). ELISA experiments were performed in strict accordance with the manufacturers' instructions.

\section{Reverse transcription polymerase chain reaction (qRT-PCR)}

Total RNA was extracted from HepG2 cells and liver biopsy using Trizol reagent from Invitrogen (Thermo Fisher, catalog number: 15596026). cDNA synthesis was performed using Maxima Universal First Strand cDNA Synthesis Kit from Thermo Scientific (catalog number: EP0742). qRT-PCR reactions were performed with FastStart Universal SYBR Green Master (Rox) from Roche (catalog number: 04913850001). The experiments were performed according to the manufacturer's instructions. The sequences of the primers used for qRT-PCR analyses were listed in Table 1.

Table 1

Primers of OLFM4 and HIF1a.

\begin{tabular}{|lllll|}
\hline Gene & Primer type & sequence & Tm & Product size \\
\hline \multirow{2}{*}{ OLFM4 } & Sense & TCAGCAAACCGTCTGTGGTT & 60.11 & 70 \\
\cline { 2 - 4 } & Anti-sense & TCCCTACCCCAAGCACCATA & 59.95 & \\
\multirow{2}{*}{ HIF1a } & Sense & GTCTGAGGGGACAGGAGGAT & 60.03 & 80 \\
\cline { 2 - 4 } & Anti-sense & CTCCTCAGGTGGCTTGTCAG & 60.04 & \\
\cline { 2 - 4 } & & & \multicolumn{2}{|c}{} \\
\hline
\end{tabular}

\section{Immunohistochemical staining (IHC)}

For IHC analysis, liver cancer and paracancerous tissues from liver patients were formalin-fixed, followed by being embedded using paraffin method. Then, paraffin blocks were prepared into slides, followed by IHC process using standard instructions. Slides were probed with anti-OLFM4 antibody (ab10586, Abcam1), followed by were visualized using $D A B+$ as a chromogen.

\section{Cell culture and transfection}


Human hepatocellular carcinoma (HepG2) cells were purchased from ATCC (ATCC HB-8065). HepG2 cells were maintained in Dulbecco modified Eagle medium (DMEM, catalog number: 11965118) containing 10\% fetal bovine serum (Gibco, catalog number: 10099141C), 100 units $/ \mathrm{ml}$ penicillin, and $0.1 \%(\mathrm{w} / \mathrm{v}$ ) streptomycin (catalog number: 15140163 ) at $37^{\circ} \mathrm{C}$ in a humidified atmosphere of $5 \% \mathrm{CO} 2$.

SiRNAs against OLFM4 and HIF-1a against OLFM4 were synthesized by ThermoFisher Scientific. The sequences of siRNAs were listed in Table 2. The full-length coding sequence of OLFM4 was cloned into pcDNA3.1 vector (Yuanjing Biotechnology, Guangzhou, Guangdong, China). For transfection, siRNAs or plasmids were transfected in HepG2 cells using Lipofectamine 3000 (ThermoFisher Scientific, catalog number: L3000001) according to the manufacturer's instructions.

Table 2

sequences of siRNAs.

\begin{tabular}{|lllll|}
\hline Genes & siRnA & sequence & GC\% \\
\hline & OLFM4 & OLFM4\#1 & AAGACCAAGCTGAAAGAGTGT & 42.86 \\
\cline { 2 - 5 } & OLFM4\#1 & AAGGATACCATTTCTTACACT & 33.33 \\
\hline HIF1a & HIF1a\#1 & AAGGATGCAAATCTAGTGAAC & 38.10 \\
\cline { 3 - 5 } & HIF1a\#2 & AAGGACAAGTCACCACAGGAC & 52.38 \\
\hline
\end{tabular}

\section{Western blotting}

It was used the conventional protocol for Western blotting (WB). Briefly, cells were lysed for total protein isolation using RIPA lysis buffer (Beyotime, catalog: P0013B). The protein concentration was determined using the Bradford assay. Equal amounts of total protein were separated by SDS-PAGE electrophoresis, transferred to PVDF membranes, and blocked with 5\% skim milk powder at room temperature for $1 \mathrm{~h}$. Next, the PVDF membranes were washed with TBST containing $\mathrm{NaCl}$, Tris- $\mathrm{HCl}$, and Tween-20 and incubated with primary antibodies against target proteins including OLFM4 (Abcam, catalog number: ab267805) and $\beta$-actin (Abcam, ab8226) at $4^{\circ} \mathrm{C}$ overnight, followed by two washes with TBST. Then, membranes were incubated with the appropriate secondary antibodies at room temperature for $1 \mathrm{~h}$ and washed three times with TBST. Protein bands were visualized by chemiluminescence (BeyoECL Plus, Beyotime, P0018S, Shanghai, China).

\section{Statistical analysis}

Tests to examine the differences between groups included Student's $t$ test and one-way ANOVA. The levels of mRNA expression between cancer tissues and normal tissues were analysed by t-test. All analyses related to patient survival were tested by Kaplan-Meier survival analysis (log-rank method). A p $<0.05$ was regarded as statistically significant.

\section{Results}




\section{Patient Characteristics}

The clinical characteristics of the100 liver cancer patients were presented in Table 3, including age, gender, BMI (body mass index), hepatitis infections, NASH status, OLFM4 blood levels, and tumor size. It is indicated that there are 62 females and 58 males; the age of male patients is $61.87 \pm 1.40$, while the age of female patients is $56.75 \pm 2.37(P=0.0778)$; in female patients, $B M I$ is $20.67 \pm 0.27$, while $B M I$ is $20.49 \pm 1.86$ for male patients $(P=0.4683)$; there are $20 \mathrm{HBV}$ infected female patients, and $31 \mathrm{HBV}$ infected male patients $(P=0.895)$; there are $6 \mathrm{HCV}$ infected female patients, and $7 \mathrm{HCV}$ infected male patients $(P=0.668)$; there are $5 \mathrm{NASH}$ female patients, and $18 \mathrm{NASH}$ male patients $(P=0.059)$; for OLFM4 level in blood, female patients are $44.06 \pm 1.67(\mathrm{U} / \mathrm{L})$, and male patients are $43.16 \pm 2.23(\mathrm{U} / \mathrm{L})(\mathrm{P}$ $=0.7815)$; in terms of tumor size, female patients are $2.683 \pm 0.30$ ( $\mathrm{mm}$, diameter), and male patients are $43.16 \pm 2.23$ ( $\mathrm{mm}$, diameter) $(P=1.72 \pm 0.14)$.

Table 3

basic physiological characteristics of liver cancer.

\begin{tabular}{|c|c|c|c|c|}
\hline & & Female & Male & $P$ value \\
\hline Patients (n) & & 38 & 62 & \\
\hline Age (years; means \pm SEM) & & $61.87 \pm 1.40$ & $56.75 \pm 2.37$ & 0.0778 \\
\hline BMI & & $20.67 \pm 0.27$ & $20.49 \pm 1.86$ & 0.4683 \\
\hline \multirow[t]{2}{*}{ Hepatitis infections } & $\mathrm{HBV}+$ & 20 & 31 & 0.895 \\
\hline & $\mathrm{HCV}+$ & 6 & 17 & 0.668 \\
\hline $\mathrm{NASH}$ & & 5 & 18 & 0.059 \\
\hline OLFM4 blood levels (U/L) (mean \pm SEM) & & $44.06 \pm 1.67$ & $43.16 \pm 2.23$ & 0.7815 \\
\hline Tumor size (mm, diameter) (means \pm SEM) & & $2.683 \pm 0.30$ & $1.72 \pm 0.14$ & 0.7760 \\
\hline
\end{tabular}

\section{OLFM4 expression in blood and tissues}

To test the potential of OLFM4 as a biomarker of liver cancer, OLFM4 blood level was measured using ELISA, which indicated that OLFM4 blood level in liver cancer patients was significantly higher than in healthy individuals (Fig. 1, P< 0.001). To further demonstrate, mRNA expression level of OLFM4 in liver paracancerous and cancer tissues was measured, which indicated that mRNA expression level of OLFM4 in liver cancer tissues was remarkably higher than liver paracancerous tissues (Fig. 2A, $P<0.001$ ). OLFM4 staining was observed in liver cancer tissues but not in liver paracancerous tissues (Fig. 2B, $P<0.001$ ). Taken together, OLFM4 expression level is significantly up-regulated in liver cancer patients. 


\section{Diagnostic capability of OLFM4 expression and correlation with tumor size}

The receiver operating characteristic (ROC) analysis was performed to determine the diagnostic value of OLFM4 expression for liver cancer (Fig. 3). It indicated that both OLFM4 blood level (Fig. 3A) and mRNA expression level in liver tissues (Fig. 3B) had excellent diagnostic value overall, manifesting the AUCs were $0.9292(P<0.0001)$ and $0.8844(P<0.0001)$, respectively.

To further assess the diagnostic value of OLFM4 for liver cancer, the correlation between OLFM4 expression and tumor size was analyzed. As shown in Fig. 4, BMI had no clear correlation to tumor size (Fig. 4A). Interesting, OLFM4 blood level has significant correlation to tumor size $\left(R^{2}=0.4646, P<0.0001\right)$ (Fig. 4B). OLFM4 mRNA expression in liver cancer tissue has significant correlation to tumor size $\left(\mathrm{R}^{2}=\right.$ $0.5113, \mathrm{P}<0.0001$ ) (Fig. 4C). Collectively, OLFM4 has a diagnostic value to predict liver cancer, and it significantly correlated to tumor size.

\section{OLF4M closely regulated proliferation of HepG2 cells}

To investigate effects of OLFM4 on liver cancer, a human liver carcinoma cell line (e.g. HepG2) was used. First of all, two siRNAs against OLFM4 were constructed and both siRNAs showed knockdown effects on OLFM4 in HepG2 cells, while second siRNA showed better knockdown efficient (Fig. 5A). Knockdown was further verified by WB assay (Fig. 5B). Effects of siRNAs against OLFM4 on hepG2 proliferation were measured using CCK8 assay, which indicated that two siRNAs significantly reduced HepG2 proliferation (Fig. 5C). In parallel, OLFM4 over-expression plasmids were constructed, which indicated significantly regulation of OLFM4 gene in HepG2 cells after transfection (Fig. 5D). WB assay confirmed overexpression in HepG2 cells (Fig. 5E). Moreover, OLFM4 over-expression significantly promoted cell proliferation in HepG2 cells (Fig. 5F). Collectively, it is demonstrated that OLFM4 closely regulates liver cancer cell proliferation.

\section{HIF-1a involved in the regulation of OLFM4 on liver cancer}

HIF-1a was found to be the downstream gene of OLFM4, which encouraged to study whether HIF-1a involves in the regulation of OLFM4 on liver cancer. To this aim, two siRNAs against HIF-1a were synthesized and transfected in HepG2 cells, which indicated a significant knockdown (Fig. 6A). Importantly, it was found that knockdown of HIF-1a significantly alleviated promotion of OLFM4 on HepG2 proliferation (Fig. 6B). Thus, we confirmed that HIF-1a involves the regulation of OLFM4 on liver cancer cell proliferation.

\section{Discussion}


Liver cancer has been become one of most severe cancers causing a large number population dead in the world [20]. Although several therapies including chemotherapy, arterial embolization, surgical resection, and radiofrequency ablation have been developed to treat the diseases, while none of them is ideal due to native side effects [3]. Especially, the pathogenesis of liver cancer is not yet completely understood. Noninvasive diagnosis for liver cancers is wanted due to conventional method such as liver biopsy may cause significant morbidity [21]. In the present study, we first measured OLFM4 blood level in liver cancer patients and healthy controls, and mRNA expression in liver paracancerous and cancer tissues. It was found liver cancer patients had higher OLFM4 blood level, and liver cancer tissues had higher OLFM4 expression level than liver paracancerous tissues. ROC analysis indicated that OLFM4 had a high diagnostic value for liver cancer. OLFM4 had a strong correlation to tumor size. Last but not least, we confirmed that OLFM4 contributed to liver cancer cell proliferation in HepG2 cells, and HIF-1a involves in regulation of OLFM4 on liver cancer cell proliferation.

Incidence of cancers is often different in different gender. For example, it was found that gender differences existed in cancer-associated venous thromboembolism [22]. Yang et al found that females and males had different colorectal cancer survival [23]. In lung cancer, it was found that long-time survival post curative resection in early stage non-small-cell lung cancer in women is better than in men, and women showed often more molecular changes than men [24]. However, in our study, we did found gender differences for OLFM4 blood level and tumor size in liver cancer (Table 3). Whether gender is different in liver cancer needs further discussion since our number of patients in our cohort is limited.

Liver cancer has been found to induce the expression modification of a large body of genes. Zhang et al used bioinformatics analysis to identifying several key genes and pathways in hepatocellular carcinoma including GMPS, ACACA, ALB, TGFB1, KRAS, ERBB2, BCL2, EGFR, STAT3, and CD8A [25]. Similarly, Shen et al also found the expression level of a panel of genes such as TOP2A, NDC80, FOXM1, HMMR, KNTC1, PTTG1, FEN1, RFC4, SMC4, and PRC1 was significantly changed in hepatocellular carcinoma [26]. These genes might be potential non-invasive biomarkers for diagnosis of liver cancer. Pan et al found that SLC25A11 was downregulated in liver cancer compared to normal controls and low expression of SLC25A11 was significantly associated with clinical stage, vital status, histologic grade, overall survival (OS) and relapse-free survival (RFS), thus SLC25A11 may serve as a prognostic marker for liver cancer [27]. OLFM4 is generally considered as a marker o stem cells. Interestingly, Suzuki et al found OLFM4 expression was associated with nodal metastases in esophageal adenocarcinoma, and might be an informative marker with the potential to improve preoperative assessment in patients with esophageal adenocarcinoma [10]. Van der Flier found that OLFM4 was a robust marker for stem cells in human intestine and marks a subset of colorectal cancer cells [28]. Myama et al found that OLFM4, LY6D and S100A7 could be potent markers for distant metastasis in estrogen receptor-positive breast carcinoma [29]. Consistently, we found that blood level of OLFM4 was higher in liver cancer patients compared to healthy control, and mRNA expression was higher in liver cancer tissues than liver paracancerous tissues (Figs. 1 and 2). We also found that OLFM4 has a high predictive value for diagnosing liver cancer and closely correlated to tumor size (Figs. 3 and 4). However, Clemmensen et al analyzed the plasma levels of OLFM4 in normals and patients with gastrointestinal cancer, while there was no association being found 
between blood level of OLFM4 and colorectal malignancies [13]. Therefore, OLFM4 might be potential non-invasive biomarkers for several cancer types including liver cancer, while more clinical and experimental data should be accumulated to further verify the predictive capacity for liver cancers.

Seeking a therapeutic target is very important for developing effective drugs to treat cancers. In the present study, it was found that OLFM4 promoted liver cancer cell proliferation (Fig. 5). In gastric cancer cells, it was found that depletion of OLFM4 gene inhibited cell growth and increased sensitization to hydrogen peroxide and TNFa induced apoptosis [30]. Consistent with our results, Ashizawa et al reported that OLFM4 could activate STAT3 and affiliate tumor progression by decreasing expression level of GRIM19 in human hepatocellular carcinoma [31]. Interestingly, Gao et al demonstrated that HIF-1a involved in the regulation of OLFM4 on hypoxia-induced invasion, epithelial-mesenchymal transition, and chemotherapy resistance in non-small-cell lung cancer [17]. In the present study, we also confirmed that HIF-1a involved in the regulation of OLFM4 on liver cancer cell proliferation (Fig. 6). Thus, OLFM4/HIF-1a axis might be a target signaling pathway for developing novel drugs to treat liver cancer.

In summary, OLFM4 blood level is higher in liver cancer patients than in healthy individuals and mRNA expression level in liver cancer tissue than in liver paracancerous tissues. OLFM4 has high predictive capacity as a biomarker for liver cancer and closely correlated to tumor size. Importantly, it is confirmed that OLFM4 contributes to cancer cell proliferation, and HIF-1 a involves in this activity. We believe that OLFM4/HIF-1a axis might be a target signaling pathway for developing novel drugs to treat liver cancer.

\section{Declarations}

\section{Acknowledgement}

There is no funding to sponsor the study.

\section{Author contributions}

Conceptualization by Y.W. and Q.S.; data curation by F.Z. and Y.T.; formal analysis by Y.W. and Q.S.; methodology by F.Z. and Y.T.; project administration by Y.W.; supervision by Q.S.; validation by Y.W. and Q.S.; writing-original draft by Y.W.; and, writing-review \& editing by Q.S.

\section{Conflict of interests}

All authors have no conflict of interests.

\section{Data availability statement}

The authors confirm that the data supporting the findings of this study are available within the article [and/or] its supplementary materials.

\section{References}


1. Global Burden of Disease Liver Cancer, Akinyemiju C, Abera T, Ahmed S, Alam M, Alemayohu N, Allen MA, Al-Raddadi C, Alvis-Guzman R, Amoako N, Artaman Y, Ayele A, Barac TA, Bensenor A, Berhane I, Bhutta A, Castillo-Rivas Z, Chitheer J, Choi A, Cowie JY, Dandona B, Dandona L, Dey R, Dicker S, Phuc D, Ekwueme H, Zaki DU, Fischer MS, Furst F, Hancock T, Hay J, Hotez SI, Jee P, Kasaeian SH, Khader A, Khang Y, Kumar YH, Kutz A, Larson M, Lopez H, Lunevicius A, Malekzadeh R, McAlinden R, Meier C, Mendoza T, Mokdad W, Moradi-Lakeh A, Nagel M, Nguyen G, Nguyen Q, Ogbo G, Patton F, Pereira G, Pourmalek DM, Qorbani F, Radfar M, Roshandel A, Salomon G, Sanabria JA, Sartorius J, Satpathy B, Sawhney M, Sepanlou M, Shackelford S, Shore K, Sun H, Mengistu J, Topor-Madry DT, Tran R, Ukwaja B, Vlassov KN, Vollset V, Vos SE, Wakayo T, Weiderpass T, Werdecker E, Yonemoto A, Younis N, Yu M, Zaidi C, Zhu Z, Murray L, C. J. L.; Naghavi M, Fitzmaurice C, The Burden of Primary Liver Cancer and Underlying Etiologies From 1990 to 2015 at the Global, Regional, and National Level: Results From the Global Burden of Disease Study 2015. JAMA Onco/ 2017, 3, (12), 1683-1691

2. Yamashita T, Kaneko S, [Liver Cancer]. Rinsho Byori 2016, 64, (7), 787-796

3. Sun JH, Luo Q, Liu LL, Song GB (2016) Liver cancer stem cell markers: Progression and therapeutic implications. World J Gastroenterol 22(13):3547-3557

4. Li L, Wang H, Heterogeneity of liver cancer and personalized therapy. Cancer Lett 2016, 379, (2), $191-7$

5. Raoul JL, Forner A, Bolondi L, Cheung TT, Kloeckner R, de Baere T (2019) Updated use of TACE for hepatocellular carcinoma treatment: How and when to use it based on clinical evidence. Cancer Treat Rev 72:28-36

6. Anwanwan D, Singh SK, Singh S, Saikam V, Singh R (2020) Challenges in liver cancer and possible treatment approaches. Biochim Biophys Acta Rev Cancer 1873(1):188314

7. Valo I, Raro P, Boissard A, Maarouf A, Jezequel P, Verriele V, Campone M, Coqueret O, Guette C (2019) OLFM4 Expression in Ductal Carcinoma In Situ and in Invasive Breast Cancer Cohorts by a SWATHBased Proteomic Approach. Proteomics 19(21-22):e1800446

8. Liu W, Rodgers GP (2016) Olfactomedin 4 expression and functions in innate immunity, inflammation, and cancer. Cancer Metastasis Rev 35(2):201-212

9. Wang XY, Chen SH, Zhang YN, Xu CF (2018) Olfactomedin-4 in digestive diseases: A mini-review. World J Gastroenterol 24(17):1881-1887

10. Suzuki L, Ten Kate FJC, Gotink AW, Stoop H, Doukas M, Nieboer D, Spaander MCW, van Lanschot JJB, van Wijnhoven BPL, Koch AD, Bruno MJ, Looijenga LHJ, Biermann K, Olfactomedin 4 (OLFM4) expression is associated with nodal metastases in esophageal adenocarcinoma. PLoS One 2019, 14, (7), e0219494

11. Jang BG, Lee BL, Kim WH, Olfactomedin-related proteins 4 (OLFM4) expression is involved in early gastric carcinogenesis and of prognostic significance in advanced gastric cancer. Virchows Arch 2015, 467, (3), $285-94$

12. Li J, Liu C, Li D, Wan M, Zhang H, Zheng X, Jie X, Zhang P, Li J, Hou H, Sun Q (2019) OLFM4 Inhibits Epithelial-Mesenchymal Transition and Metastatic Potential of Cervical Cancer Cells. Oncol Res 
27(7):763-771

13. Clemmensen SN, Glenthoj AJ, Heeboll S, Nielsen HJ, Koch C, Borregaard N (2015) Plasma levels of OLFM4 in normals and patients with gastrointestinal cancer. J Cell Mol Med 19(12):2865-2873

14. Chen X, Khalid K, Chen D, Qiu C, Serum levels of olfactomedin 4: a biomarker for asthma control state in asthmatics. Ann Transl Med 2020, 8, (7), 494

15. Su W, Luo L, Wu F, Lai Z, Li X, Xie Z, Tang Z, Yang Z, Liang R, Low expression of olfactomedin 4 correlates with poor prognosis in smoking patients with non-small cell lung cancer. Hum Pathol 2015, 46, (5), 732-8

16. Guette $C$, Valo I, Vetillard A, Coqueret $O$, Olfactomedin- 4 is a candidate biomarker of solid gastric, colorectal, pancreatic, head and neck, and prostate cancers. Proteomics Clin Appl 2015, 9, (1-2), 5863

17. Gao XZ, Wang GN, Zhao WG, Han J, Diao CY, Wang XH, Li SL, Li WC, Blocking OLFM4/HIF-1alpha axis alleviates hypoxia-induced invasion, epithelial-mesenchymal transition, and chemotherapy resistance in non-small-cell lung cancer. J Cell Physio/ 2019

18. Lin MC, Lin JJ, Hsu CL, Juan HF, Lou PJ, Huang MC, GATA3 interacts with and stabilizes HIF-1alpha to enhance cancer cell invasiveness. Oncogene 2017, 36, (30), 4380

19. Wei TT, Lin YT, Tang SP, Luo CK, Tsai CT, Shun CT, Chen CC (2020) Metabolic targeting of HIF-1alpha potentiates the therapeutic efficacy of oxaliplatin in colorectal cancer. Oncogene 39(2):414-427

20. Marengo A, Rosso C, Bugianesi E, Liver Cancer: Connections with Obesity, Fatty Liver, and Cirrhosis. Annu Rev Med 2016, 67, 103 - 17

21. Moreno C, Mueller S, Szabo G (2019) Non-invasive diagnosis and biomarkers in alcohol-related liver disease. J Hepatol 70(2):273-283

22. Riondino S, Guadagni F, Formica V, Ferroni P, Roselli M, Gender Differences in Cancer-associated Venous Thromboembolism. Curr Med Chem 2017, 24, (24), 2589-2601

23. Yang Y, Wang G, He J, Ren S, Wu F, Zhang J, Wang F (2017) Gender differences in colorectal cancer survival: A meta-analysis. Int J Cancer 141(10):1942-1949

24. Welcker K, [Gender Differences in Lung Cancer]. Zentralb/ Chir 2015, 140, (3), 260-5

25. Zhang C, Peng L, Zhang Y, Liu Z, Li W, Chen S, Li G, The identification of key genes and pathways in hepatocellular carcinoma by bioinformatics analysis of high-throughput data. Med Oncol 2017, 34, (6), 101

26. Shen S, Kong J, Qiu Y, Yang X, Wang W, Yan L (2019) Identification of core genes and outcomes in hepatocellular carcinoma by bioinformatics analysis. J Cell Biochem 120(6):10069-10081

27. Pan G, Wang R, Jia S, Li Y, Jiao Y, Liu N (2020) SLC25A11 serves as a novel prognostic biomarker in liver cancer. Sci Rep 10(1):9871

28. van der Flier LG, Haegebarth A, Stange DE, van de Wetering M, Clevers H, OLFM4 is a robust marker for stem cells in human intestine and marks a subset of colorectal cancer cells. Gastroenterology $2009,137,(1), 15-7$ 
29. Mayama A, Takagi K, Suzuki H, Sato A, Onodera Y, Miki Y, Sakurai M, Watanabe T, Sakamoto K, Yoshida R, Ishida T, Sasano H, Suzuki T, OLFM4, LY6D and S100A7 as potent markers for distant metastasis in estrogen receptor-positive breast carcinoma. Cancer Sci 2018, 109, (10), 3350-3359

30. Liu RH, Yang MH, Xiang H, Bao LM, Yang HA, Yue LW, Jiang X, Ang N, Wu LY, Huang Y (2012) Depletion of OLFM4 gene inhibits cell growth and increases sensitization to hydrogen peroxide and tumor necrosis factor-alpha induced-apoptosis in gastric cancer cells. J Biomed Sci 19:38

31. Ashizawa Y, Kuboki S, Nojima H, Yoshitomi H, Furukawa K, Takayashiki T, Takano S, Miyazaki M, Ohtsuka M, OLFM4 Enhances STAT3 Activation and Promotes Tumor Progression by Inhibiting GRIM19 Expression in Human Hepatocellular Carcinoma. Hepatol Commun 2019, 3, (7), 954-970

Figures

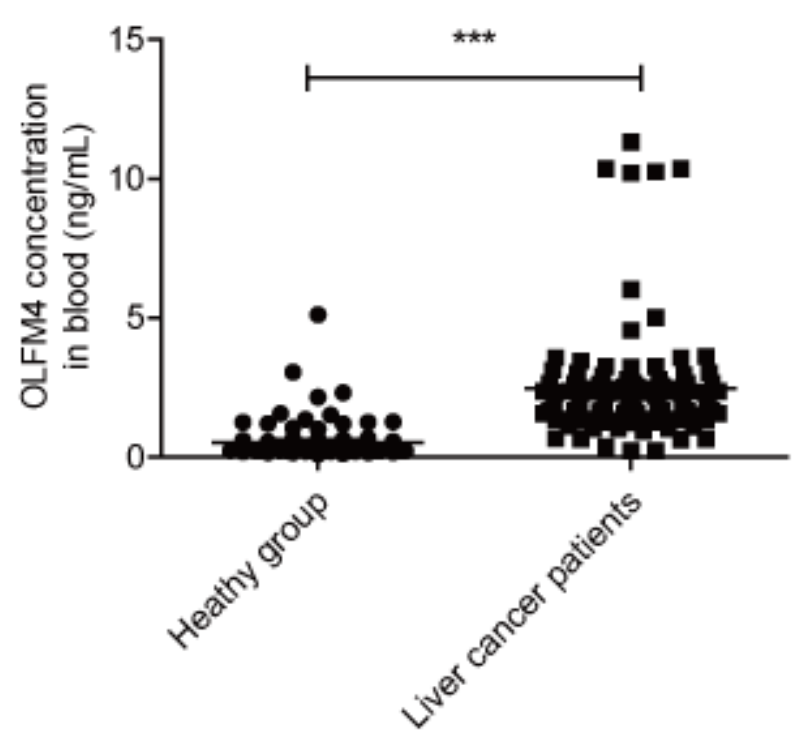

Figure 1

The blood level of OLFM4 in healthy individuals and liver cancer patients. ${ }^{\star \star *} \mathrm{P}<0.001$. 
A

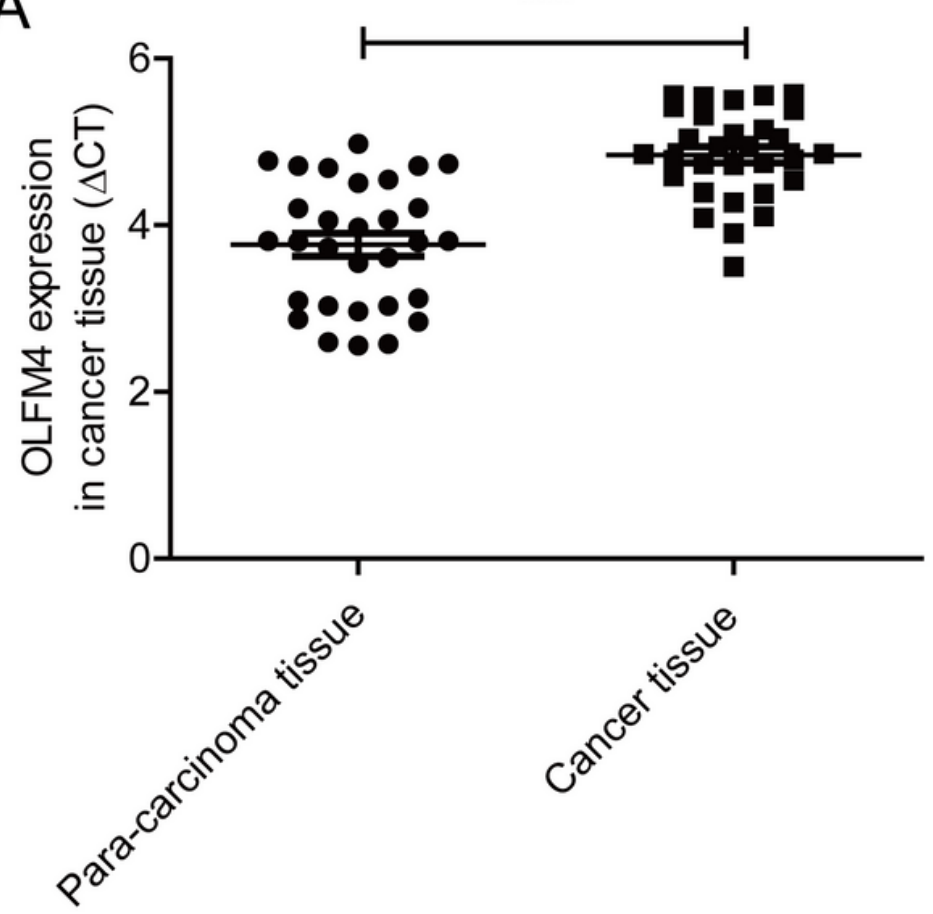

B
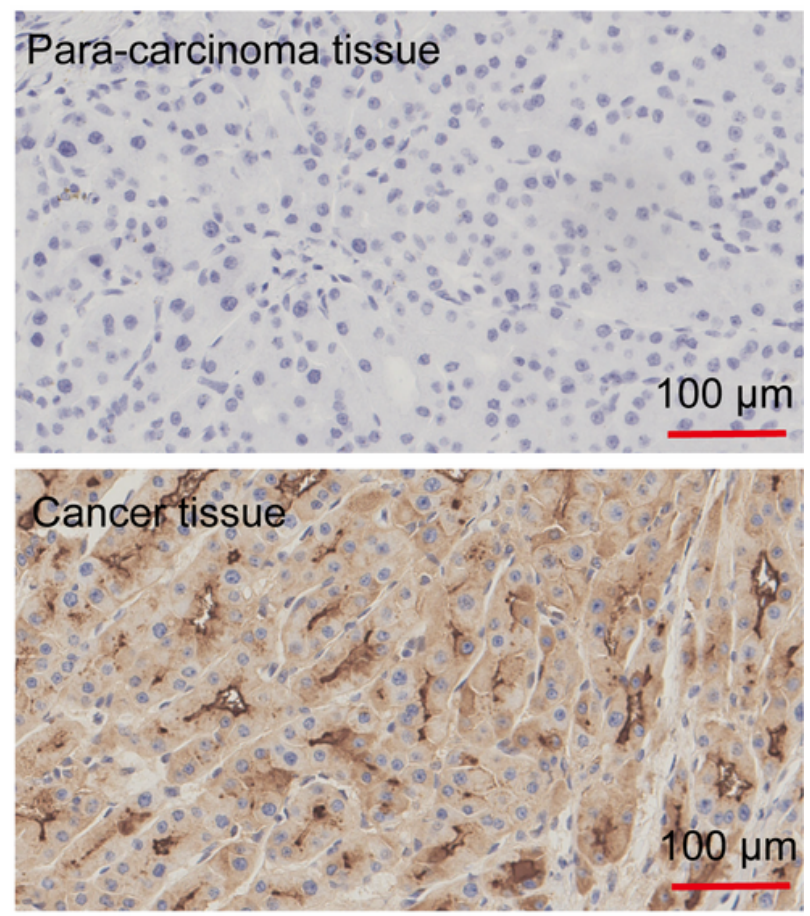

Figure 2

mRNA and protein levels of OLFM4 in para-carcinoma tissue and cancer tissue in liver cancer patients. (A) mRNA expression of OLFM4 in para-carcinoma tissue and cancer tissue in liver cancer patients measured by qRT-PCR assay, ${ }^{* *} \mathrm{P}<0.001$; (B) protein levels of OLFM4 in para-carcinoma tissue and cancer tissue in liver cancer patients measured by IHC assay. 


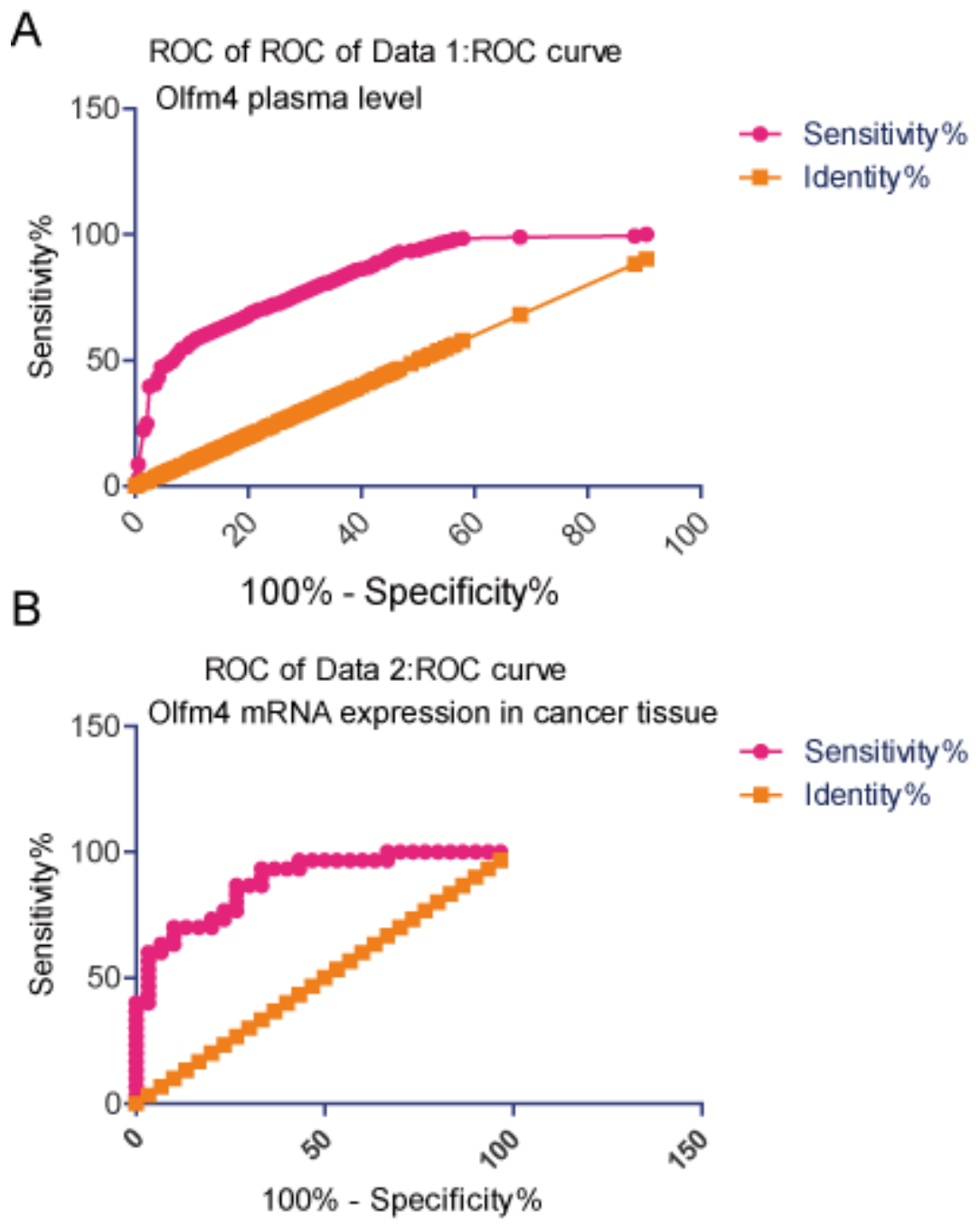

Figure 3

ROC analysis of the performance of OLFM4 expression in identification of liver cancer. (A) ROC analysis using blood level of OLFM4 to predict liver cancer; (B) ROC analysis using mRNA expression of OLFM4 in liver cancer tissue to predict liver cancer.

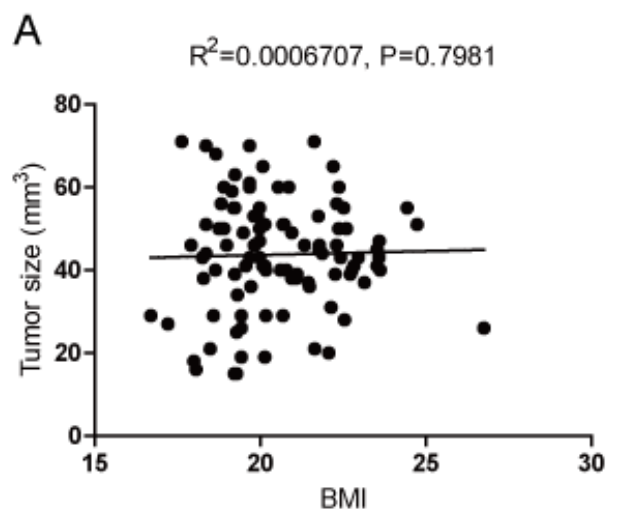

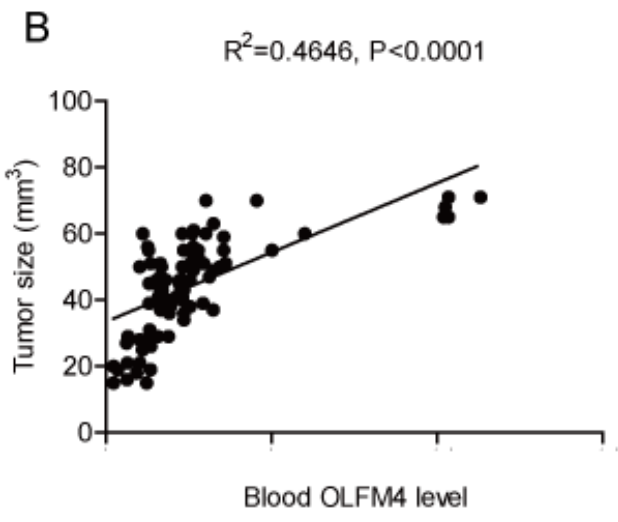

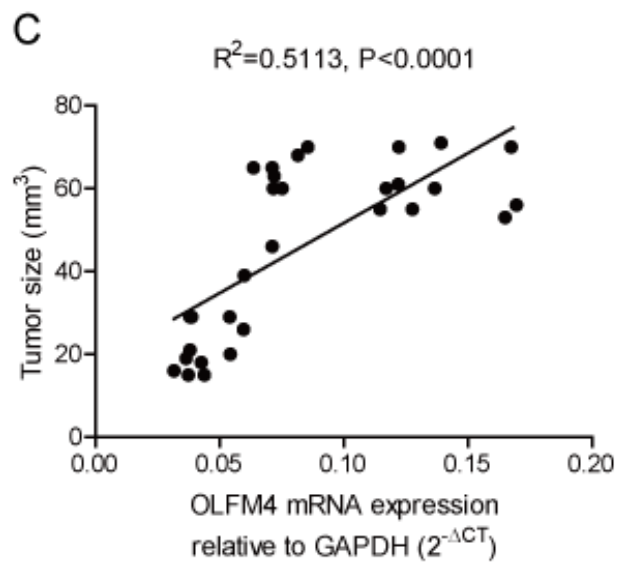

Figure 4 
Correlation of OLFM4 to tumor size. (A) correlation analysis about BMI to tumor size; (B) correlation analysis about OLFM4 blood level to tumor size; (C) correlation analysis about mRNA expression of OLFM4 in liver cancer tissue to tumor size.

A

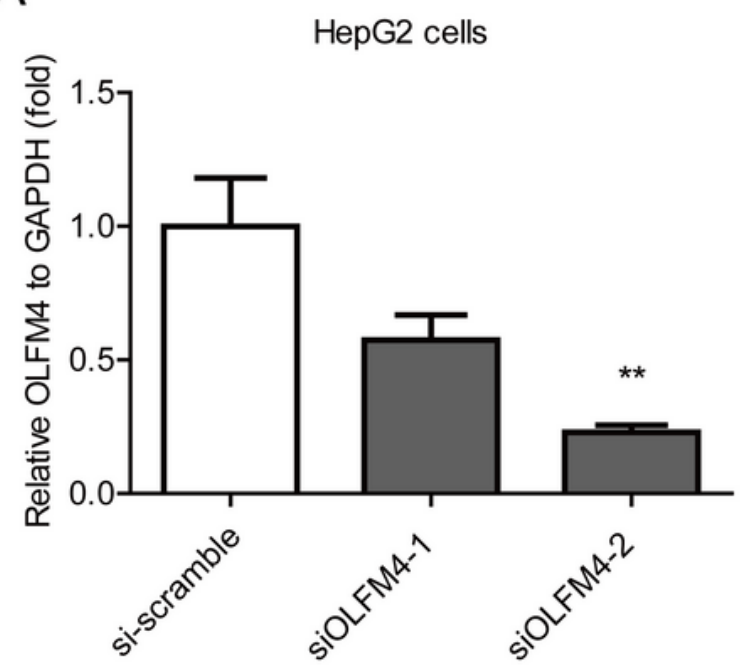

C

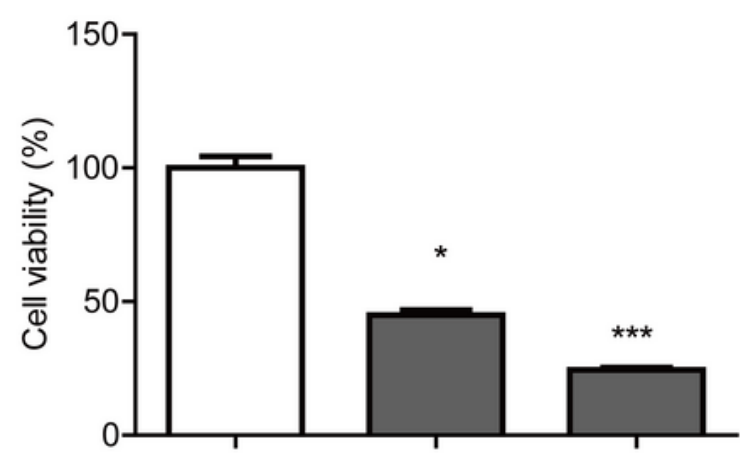

$E$
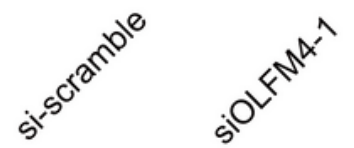

CTR OLFM4

anti-OLFM4
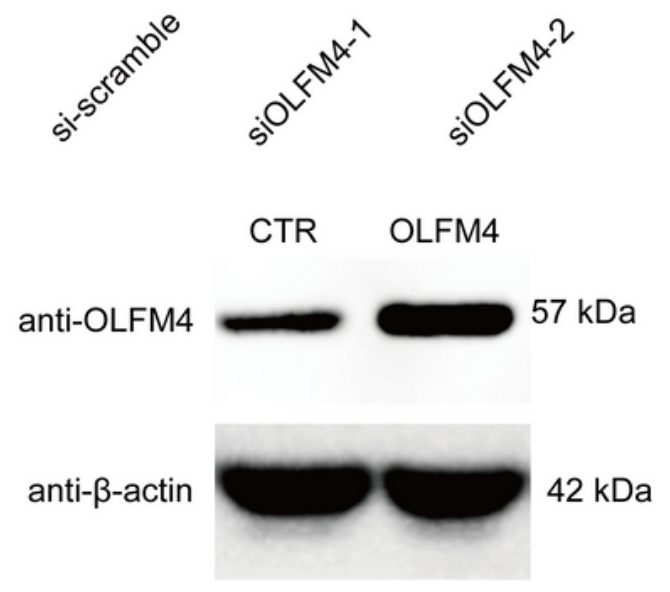

B

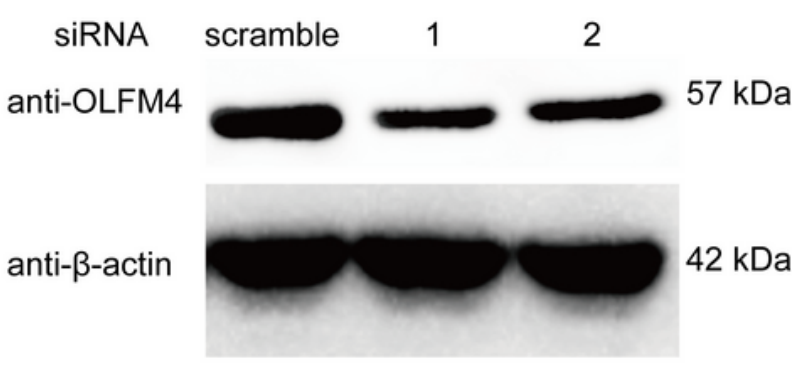

D

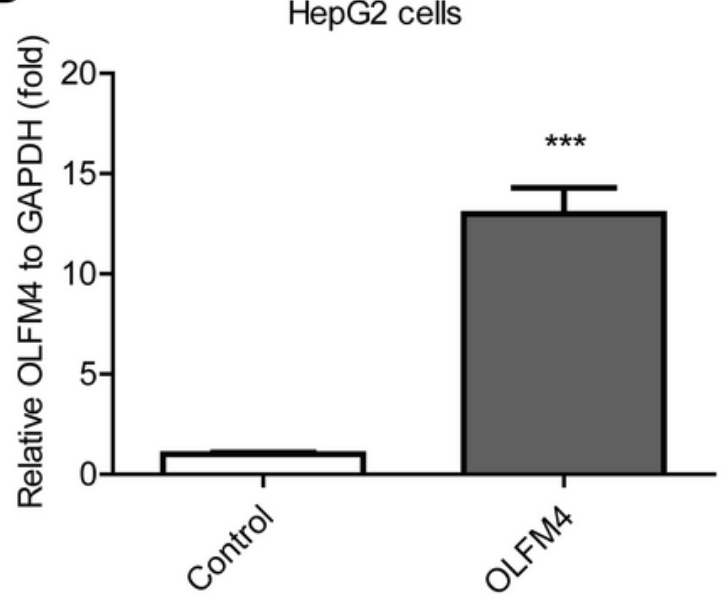

$\mathrm{F}$

HepG2 cells

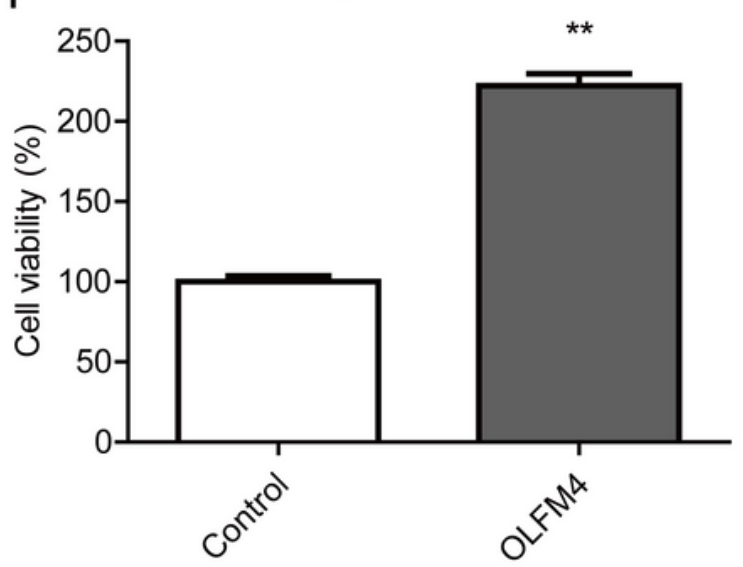

\section{Figure 5}

OLFM4 regulated liver cancer cell proliferation in HepG2 cells. (A) siRNAs against OLFM4 successfully inhibited mRNA expression of OLFM4 in HepG2 cells measured by qRT-PCR assay ( $\left.{ }^{*} \mathrm{P}<0.01\right)$; (B) siRNAs 
against OLFM4 successfully inhibited protein level of OLFM4 in HepG2 cells measured by western blot assay; (C) siRNAs against OLFM4 could inhibit proliferation of HepG2 cells ( ${ }^{*} P<0.05,{ }^{*} \mathrm{P}<0.01$ ); (D) OLFM4 plasmid transfection could over-express the gene in HepG2 cells detected by qRT-PCR assay ( $\left.{ }^{* *} \mathrm{P}<0.001\right)$; (E) OLFM4 plasmid transfection could up-regulate the protein level of OLFM4 in HepG2 cells detected by western blot assay; (F) OLFM4 plasmid could affiliate proliferation of HepG2 cells $(* * P<0.01)$.

A

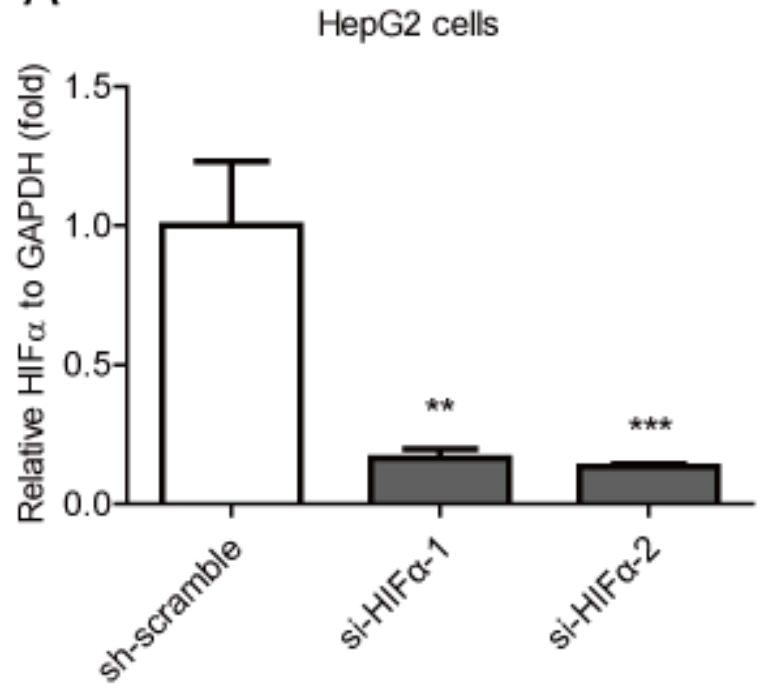

B

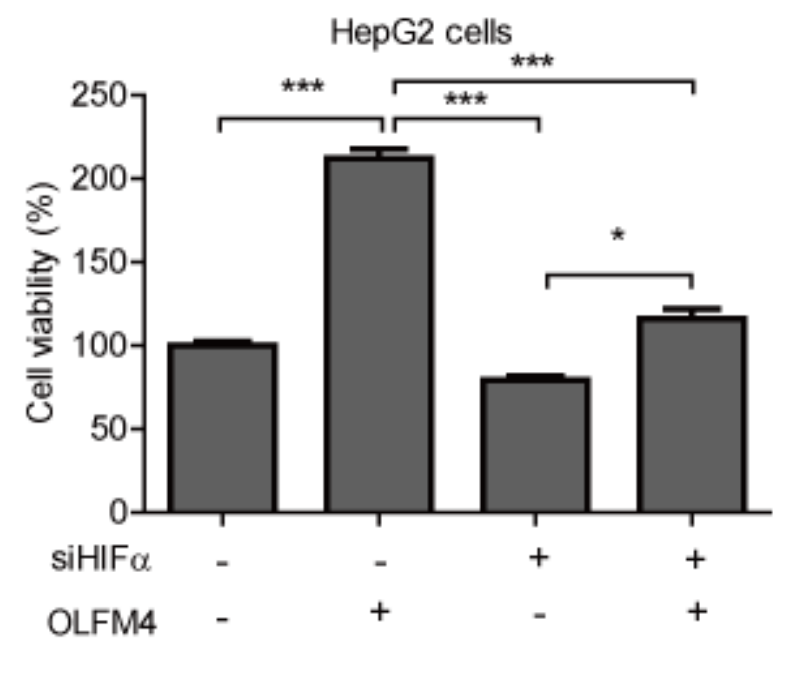

\section{Figure 6}

HIF1a involved in regulation of OLFM4 on liver cancer proliferation. (A) siRNAs against HIF1a successfully inhibited mRNA expression of HIF1a in HepG2 cells measured by qRT-PCR assay $\left({ }^{*} P<0.01\right.$, $\star \star * P<0.001)$; (B) the knockdown of HIF1 a alleviated increase of OLFM4 plasmid on liver cancer proliferation in HepG2 cells. 